\title{
Influencia del tamaño de partícula en la extracción de ácidos húmicos de carbón bituminoso
}

\section{Influence of particle size in the extraction of bituminous carbon humic acids}

\author{
Mayerlis Vásquez Osorio ${ }^{1}$; Fredy Colpas Castillo²; Arnulfo Tarón Dunoyer ${ }^{3}$
}

\begin{abstract}
${ }^{1}$ Ing. Químico, M.Sc., Docente asociado, Facultad de diseño e ingeniería. Fundación Tecnológica Antonio de Arévalo -TECNAR. Avenida Pedro de Heredia Calle 49A \# 31-45, Sector Tesca, Cartagena, Colombia, e-mail: mayerlis.vasquez@tecnar.edu.co, Dhttps://orcid.org/0000-0002-4423-4955

${ }^{2}$ Ing. Químico, M.Sc., Profesor investigador, Facultad de Ciencias Exactas y Naturales, Departamento de Química, Grupo de investigación Carboquímica. Universidad de Cartagena. Campus de San Pablo, Cartagena - Bolívar, Colombia, e-mail:fcolpasc1@unicartagena.edu.co, Dhttp://orcid.org/0000-00022262-870X
\end{abstract}

${ }^{3}$ Químico Farmacéutico, M.Sc., Profesor investigador, Facultad de Ingenierías, Grupo de GIBAE. Universidad de Cartagena. Av del Consulado Calle 30 \# $48-$ 152, Cartagena - Bolívar, Colombia, e-mail: atarond@unicartagena.edu.co, Dhttp://orcid.org/0000-0002-6942-4480

Cómo citar: Vásquez Osorio, M.; Colpas Castillo, F.; Tarón Dunoyer, A. 2019. Influencia del tamaño de partícula en la extracción de ácidos húmicos de carbón bituminoso. Rev. U.D.C.A Act. \& Div. Cient. 22(1):e1209. https://doi.org/10.31910/rudca.v22.n1.2019.1209

Artículo de acceso abierto publicado por Revista U.D.C.A Actualidad \& Divulgación Científica, bajo una licencia Creative Commons CC BY-NC 4.0

Recibido: Mayo 23 de 2018

Aceptado: Abril 30 de 2019

\section{RESUMEN}

Las sustancias húmicas son un conjunto de macromoléculas provenientes de materia orgánica biodegradada. Estas sustancias, se pueden obtener de carbones de alto rango y son importantes en la eliminación de contaminantes en soluciones acuosas. En esta investigación, se evaluó la influencia del tamaño de partículas en la extracción de ácidos húmicos, a partir de muestras de carbón bituminoso. Se realizó una oxidación en solución acuosa de las muestras con peróxido de hidrógeno, a concentraciones de 50, 40 y $30 \%$, respectivamente, tiempos de extracción de 3 y 6 horas y tamaño de partícula a número de malla 60 y 120 . Se caracterizaron las muestras de carbón por espectrometría infrarroja (FTIR). Se realizó un análisis próximo, para determinar $\%$ de humedad, $\%$ de materia volátil (\%MV), \% de ceniza, $\%$ de carbono fijo (\%CF) y relación $\% \mathrm{CF} / \mathrm{MV}$. El análisis termogravimétrico (TGA), se realizó en equipo de termografía TGA Q-500. El mayor porcentaje de extracción $(76,8 \pm 3,50)$, se obtuvo a una concentración de $50 \%$ de agente oxidante, tiempo de extracción de 6 horas y tamaño de partícula a malla No. 120. El tiempo de extracción influye en la cantidad de ácido húmico que se puede extraer, una concentración de 40\% de agente oxidante; no se encontraron diferencias estadísticamente significativas $(\mathrm{P}>0,05)$, para tiempos de $3 \mathrm{~h}(32,8 \pm 1,0$, malla 120) y 3 h $(30,6 \pm 2,4$, malla 60). La materia carbonácea utilizada en esta investigación puede ser un candidato potencial para obtener ácidos húmicos útiles en la industria agrícola.

Palabras clave: ácidos húmicos; carbón bituminoso; extracción; fertilizante; oxidación (Tesauro agrícola).

\section{ABSTRACT}

Humic substances are a group of macromolecules from biodegradable organic matter. These substances can be obtained from high-rank coals and are important in the removal of contaminants in aqueous solutions. In this study, the influence of particle size on the extraction of humic acids from samples of bituminous coal is evaluated. Oxidation in aqueous solution of the samples with hydrogen peroxide 
was carried out at concentrations of 50, 40 and 30\% respectively, extraction times of 3 and 6 hours and particle size at 60 and 120 mesh number. The carbon samples were characterized by infrared spectrometry (FTIR). A close analysis was performed to determine $\%$ moisture, $\%$ volatile matter ( $\%$ MV), $\%$ ash, $\%$ fixed carbon $(\%$ $\mathrm{CF}$ ) and $\% \mathrm{CF} / \mathrm{MV}$ ratio. Thermogravimetric analysis (TGA) was performed on TGA Q-500 thermography equipment. The highest percentage of extraction $(76.8 \pm 3.50)$ was obtained at a concentration of $50 \%$ of oxidizing agent, extraction time of 6 hours and particle size to mesh No 120. The extraction time influences the amount of humic acid that can be extracted, a concentration of $40 \%$ of oxidizing agent, no statistically significant differences were found ( $\mathrm{P}>$ $0.05)$ for times of $3 \mathrm{~h}$ (32.8 $\pm 1.0,120 \mathrm{mesh})$ and $(30.6 \pm 2.4,60 \mathrm{mesh})$. The carbonaceous material used in this research may be a potential candidate for obtaining useful humic acids in the agricultural industry.

Keywords: agricultural fertilizers; carbonaceous matter; humic substances; oxidation (Agricultural Thesaurus).

\section{INTRODUCCIÓN}

En las sustancias húmicas, se encuentra una fracción denominada ácidos húmicos, que poseen anillos aromáticos y estructuras poliaromáticas, enlazados por medio de cadenas alifáticas. Los grupos carboxílicos presentes en la estructura de estos ácidos son los responsables del intercambio iónico con los minerales presentes en el suelo, estimulando el crecimiento de la planta (Peña et al. 2005; Canellas \& Olivares, 2014; Pantoja Guerra et al. 2016). Constituyen uno de los componentes principales de algunos suelos, turbas y carbones. Éstas, se clasifican en ácidos húmicos (AH), ácidos fúlvicos (AF) y huminas (Canellas \& Olivares, 2014). Los ácidos húmicos son la fracción de las sustancias húmicas insolubles en soluciones acuosas bajo condiciones acidas $(\mathrm{pH}<2)$, pero sí solubles a valores mayores de $\mathrm{pH}$. La base de su estructura es altamente compleja, formada por un grupo de estructuras aromáticas y cadenas alifáticas. Poseen varios grupos funcionales, dentro de las cuales, están los hidroxilos, carboxilos, fenólicos, entre otros (Gomes de Melo et al. 2016; Fischer, 2017).

Los ácidos húmicos tienen efectos físicos, químicos y biológicos sobre el suelo, por ello, en la actualidad, su uso principal es en la agricultura, como fertilizante orgánico, por las grandes ventajas que brinda, tanto al suelo como a las plantas (Pédrot et al. 2010). Otros usos, no menos importantes, se basan en su poder para transportar compuestos orgánicos no iónicos, como pesticidas y contaminantes, pudiéndolos eliminar de soluciones acuosas (Nardi et al. 2016). Además, en la industria del cemento, se pueden usar como agentes licuantes del concreto u hormigón, pues mejora sus propiedades fisco-químicas. En la industria del papel es usado para colorear el papel y mejorar el grosor. En el tratamiento de aguas residuales, se emplea para eliminar metales tóxicos (como el plomo, mercurio, níquel, zinc y cromo), grasas, aceites y materia suspendida (Lipczynska-Kochany, 2018).

La fuente, tradicionalmente usada para el aislamiento de sustancias húmicas es el suelo; sin embargo, hay otra cantidad de materiales que pueden ser usados como materia prima, donde se destacan los carbones, las turbas y el compost (Manzak et al. 2017; De Souza \& Bragança, 2017). Los modelos estructurales de los carbones bituminosos de alto rango (Barrera et al. 2014) son similares a las estructuras de los ácidos húmicos, haciendo estos minerales propicios para la extracción de sustancias húmicas. Se pueden someter a reacciones de oxidación, para incrementar los grupos oxigenados en la matriz carbonacea y, por ende, el de ácidos húmicos y, así, mayor eficiencia en la extracción de estas sustancias (Kodama et al. 2018).

En esta investigación, se evalúa la influencia de tamaño de partícula, la concentración de agente oxidante y el tiempo en la extracción de ácidos húmicos, a partir de carbón bituminoso, procedente de una mina de la región del Cerrejón, en el departamento de La Guajira (Colombia).

\section{MATERIALES Y MÉTODOS}

Diseño experimental: Se utiliza un diseño experimental de tipo factorial, con arreglo $2^{2} \mathrm{X} 3$, ensayando dos niveles de tiempo (3h y $6 \mathrm{~h})$, tres niveles de concentración de 30, 40 y 50\% de agente oxidante $\left(\mathrm{H}_{2} \mathrm{O}_{2}\right)$ y dos niveles de tamaño de partícula, de $0,25 \mathrm{~mm}$ (malla 60) y $0,125 \mathrm{~mm}$ (malla 120).

Preparación de la muestra: El carbón procedente de la mina del Cerrejón, ubicada en el departamento de La Guajira (Colombia), fue triturada en un molino de bola marca Pulvex y tamizada a dos tamaños de partícula, utilizando tamices con número de malla 60 y 120. La desmineralización y la remoción del solvente, se realizó de acuerdo con el procedimiento sugerido por Anillo-Correa et al. (2013).

Oxidación Acuosa: Se realizó según la metodología de AnilloCorrea et al. (2013).

Caracterización de los materiales: Las muestras del carbón y el ácido húmico fueron caracterizadas por espectrometría infrarroja (FTIR). Los estudios próximales, acorde con las normas ASTM (2012), para determinar el porcentaje de humedad, de materia volátil $(\% \mathrm{Mv})$, de ceniza, porcentaje de carbono fijo (\%CF) y la relación \% CF/Mv (Anillo-Correa et al. 2013; Colpas Castillo et al. 2017).

Análisis termogravimétrico: El análisis termogravimétrico (TGA), se efectuó en un equipo de termografía TGA Q-500, con acople de horno estándar, utilizando un flujo de nitrógeno de $50 \mathrm{~mL} \mathrm{~min}^{-1}$ (Manals-Cutiño et al. 2011).

Mediante la ecuación 1, se determina la cantidad de ácidos húmicos.

$$
\frac{\text { masa del Ácido Húmico }}{\text { Masa del Carbón }} \times 100 \quad \text { (Ecuación 1) }
$$

Análisis estadístico: Los resultados encontrados fueron analizados estadísticamente mediante un ANOVA, utilizando el software SPSS (versión 17.0). Todos los ensayos fueron realizados por triplicado. 


\section{RESULTADOS Y DISCUSIÓN}

El análisis próximal de la muestra de carbón arrojó los siguientes resultados: humedad $=5.99 \pm 0.09$; materia volátil $(\mathrm{Mv})=22,25 \pm 0,43$; cenizas $=2,714 \pm 0,076$; carbono fijo $\left(\mathrm{CF}_{\mathrm{F}}\right)=69,05 \pm 0,58$ y relación $\mathrm{CF}_{\mathrm{F}} / \mathrm{Mv}_{\mathrm{v}}=2,83 \pm 0,03$. De acuerdo con estos resultados, se puede afirmar, que corresponde a un carbón bituminoso clase B (AnilloCorrea et al. 2013).

En la figura 1, se observa la curva del termograma (TGA) de la muestra de carbón. En el intervalo comprendido entre 100 y $200^{\circ} \mathrm{C}$, se evidencia el inicio de la descomposición térmica, con

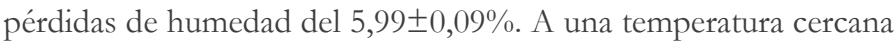
a los $200^{\circ} \mathrm{C}$, se presenta una pérdida elevada de sustancias volátiles, comportamiento que se mantiene hasta alcanzar un valor próximo a $\operatorname{los} 550^{\circ} \mathrm{C}$; a partir de esta temperatura, se puede afirmar, que comienza la principal pérdida de masa. Posteriormente, se observa un descenso regular en la pérdida de masa, que dura hasta los $630^{\circ} \mathrm{C}$, temperatura donde se inicia la formación de cenizas (De Souza \& Bragança, 2017).
En la figura 2 aparecen los espectros FTIR de la muestra de carbón antes de la oxidación. Se observa una banda ancha centrada a los $3400 \mathrm{~cm}^{-1}$, correspondiente a grupos $\mathrm{OH}$ superficiales o fenólicos de la matriz carbonácea. Las bandas analizadas en el intervalo entre $2915-2800 \mathrm{~cm}^{-1}$ son productos de los estiramientos $\mathrm{CH}$ de cadenas alifáticas presentes en la estructura del carbón; estas cadenas alifáticas, se confirman por la banda a $1380-1436 \mathrm{~cm}^{-1}$, las cuales, se atribuyen a deformaciones del ángulo del enlace $\mathrm{CH}$.

La estructura policondensada aromática de los carbones en estudio originó, de igual manera, una banda a los $1600 \mathrm{~cm}^{-1}$, ocasionada por estiramientos $\mathrm{C}=\mathrm{C}$ del tipo aromático y por las bandas entre 800$750 \mathrm{~cm}^{-1}$, que se deben a deformaciones $\mathrm{CH}$ fuera del plano (Wang et al. 2017).

La materia inorgánica presente en esta muestra genera la aparición de bandas a $1178-679-811 \mathrm{~cm}^{-1}$, correspondientes al enlace Si-O o Si-O-Al, mientras que las bandas a $537-465 \mathrm{~cm}^{-1}$, se atribuyen a enlaces $\mathrm{Fe}-\mathrm{O}$ y $\mathrm{Zn}-\mathrm{O}$, respectivamente.



Figura 1. Termograma (TGA) de la muestra de carbón sin oxidar.

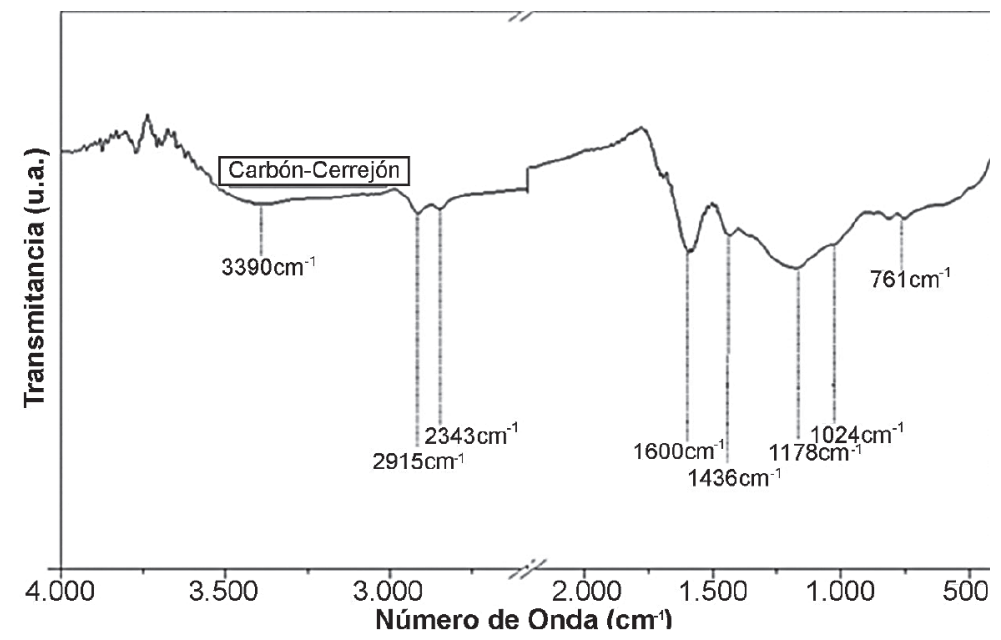

Figura 2. Espectro FTIR de la muestra de carbón sin oxidar. 
En la tabla 1, se encuentran tabulados la cantidad (\%) de ácidos húmicos extraido a diferente tamaño de partícula y concentración de agente oxidante. La mayor cantidad de AH extraídos (76,8 $\pm 3,5 \%)$ de las muestras oxidadas con $\mathrm{H}_{2} \mathrm{O}_{2}$, se obtiene al utilizar un tamaño de partícula correspondiente a un tamiz malla 120 en la matriz carbonácea, a una concentración de $50 \%$ de $\mathrm{H}_{2} \mathrm{O}_{2}$ y un tiempo de contacto con el agente oxidante de tres (6) horas. Debido al tratamiento realizado, se logra que los grupos funcionales oxigenados del peróxido de hidrógeno $\left(\mathrm{H}_{2} \mathrm{O}_{2}\right)$ sufran una descomposición, que se ve favorecida por la existencia de algunas estructuras, que son similares a los ácidos húmicos, como son los ácidos fúlvicos, entre otros (Manzak et al. 2017). En la tabla 1, se observa que una oxidación durante 6 horas provoca que la cantidad (\%) de ácidos húmicos se incremente en la estructura de los carbones a las diferentes concentraciones del agente oxidante. Este porcentaje de extracción estuvo por encima del 40\%, en la mayoría de los casos.
El mayor porcentaje de extracción, se obtuvo a partir del carbón tamizado No. 120, utilizando una concentración de $\mathrm{H}_{2} \mathrm{O}_{2}$ del $50 \%$. El contenido de ácidos húmicos en las muestras sometidas a oxidación con $\mathrm{H}_{2} \mathrm{O}_{2}$ durante 3 h fue menor a los oxidados durante 6h, pero se observó que el porcentaje de la cantidad extraída fue superior al $25 \%$, en todos los casos.

Todos valores en los porcentajes de extracción de AH, utilizando una concentración de $50 \%$ de agente oxidante y un tamaño de partículas a malla No. 120, presentan diferencias estadísticamente significativas $(\mathrm{P}<0,05)$, al compararlas con los valores obtenidos para una concentración de agente oxidante de 40 y $30 \%$, respetivamente.

El tiempo de extracción influye en la cantidad de ácido húmico que se puede extraer, aunque para una concentración de $40 \%$ de agente oxidante, no se encontraron diferencias estadísticamente

Tabla 1. Porcentajes de extracción de ácidos húmicos, utilizando diferentes concentraciones de agente oxidante, tiempos de extracción y tamaño de partículas de carbón bituminoso.

\begin{tabular}{|c|c|c|c|}
\hline \multirow{2}{*}{\begin{tabular}{c} 
Concentración de $\mathbf{H}_{2} \mathbf{O}_{2}$ \\
\multirow{2}{*}{$50 \%$}
\end{tabular}} & Tiempo & $\begin{array}{c}\text { \% de extracción AH obtenido de car- } \\
\text { bón tamizado No.120 }\end{array}$ & $\begin{array}{c}\text { \% de extracción AH obtenido de car- } \\
\text { bón tamizado No. 60 }\end{array}$ \\
\hline \multirow{2}{*}{$40 \%$} & 3 horas & $52 \pm 1,200^{\mathrm{a}}$ & $44,0 \pm 3,50^{\mathrm{b}}$ \\
\cline { 2 - 4 } & 6 horas & $76,8 \pm 2,10^{\mathrm{c}}$ & $64,4 \pm 1,86^{\mathrm{d}}$ \\
\cline { 2 - 4 } & 3 horas & $32,8 \pm 1,0^{\mathrm{ef}}$ & $30,6 \pm 2,47^{\mathrm{f}}$ \\
\hline \multirow{2}{*}{$30 \%$} & 3 horas & $64,2 \pm 3,2^{\mathrm{dg}}$ & $47,6 \pm 2,00^{\mathrm{h}}$ \\
\cline { 2 - 4 } & 6 horas & $31,60 \pm 1,8^{\mathrm{if}}$ & $25,2 \pm 1,12^{\mathrm{j}}$ \\
\hline
\end{tabular}

Valores con letras diferentes en la misma fila y en la misma columna, difieren estadísticamente $(\mathrm{p}<0,05)$. Los valores de las medidas representan la media \pm de tres determinaciones $(\mathrm{N}=3)$.

significativas $(\mathrm{P}>0,05)$, para tiempos de $3 \mathrm{~h}$, pero sí para un tamaño de partículas a malla No. 120 .

Los porcentajes de extracción de $\mathrm{AH}$ aumentaron al disminuir la concentración de peróxido de hidrógeno $\left(\mathrm{H}_{2} \mathrm{O}_{2}\right)$ y al aumentar la superficie de contacto, que se consigue al disminuir el tamaño de partícula a tamaño (tamizado a malla No. 60); en todos los casos, se encontraron diferencias estadísticamente significativas $(\mathrm{P}<0,05)$, incluso, al utilizar la misma concentración de agente oxidante, pero diferente tamaño de partícula y diferentes tiempos de extracción (Anillo-Correa et al. 2013) (Tabla 1).

Los espectros FTIR de los ácidos húmicos que se obtuvieron después de realizar la oxidación con $\mathrm{H}_{2} \mathrm{O}_{2}$, a una concentración de $50 \%$, muestran entre $3500-3200 \mathrm{~cm}^{-1}$ una absorción de tensión $\mathrm{OH}$ ancha y fuerte características de los grupos carboxílicos (Figura 3).

Esta banda es más ancha que la registrada por la muestra de carbón sin oxidar, lo que da indicio del aumento del número de grupos carboxílicos. Esta banda también aparece en los espectros obtenidos al utilizar concentraciones de agente oxidante de 40 y 30\%, diferentes tamaños de partículas y tiempos de extracción (Figuras 4 y 5). Un incremento de las bandas estrechas entre 3000 y $2800 \mathrm{~cm}^{-1}$ son producto de la tensión $\mathrm{C}-\mathrm{H}$ en las estructuras alifáticas, lo que explica que la oxidación con $\mathrm{H}_{2} \mathrm{O}_{2}$ produjo un rompimiento en los enlaces de las macroestructuras, que originaron la presencia de estos grupos alifáticos, que se pueden presentar como sustituyentes en las estructuras aromáticas o también pueden actuar como puentes (Quintero \& Bonillas, 1991).

Entre los 1800-1600 cm 1, se pueden observar las generadas por las tensiones $\mathrm{C}=\mathrm{O}$, centradas en $1270 \mathrm{~cm}^{-1}$, por la presencia de $\mathrm{C}=\mathrm{C}$ o Ar-O-R y en el intervalo de $1300-1000 \mathrm{~cm}^{-1}$, por los enlaces C-O-C. Estos resultados corresponden con la estructura molecular de los ácidos húmicos, reportados en la literatura (López et al. 2014; Izquierdo et al. 2004).

Las aguas residuales que se originan en el proceso de extracción son ricas en sustancias altamente contaminantes, como el hidróxido de sodio y el peróxido de hidrógeno. A nivel industrial, estos efluentes 

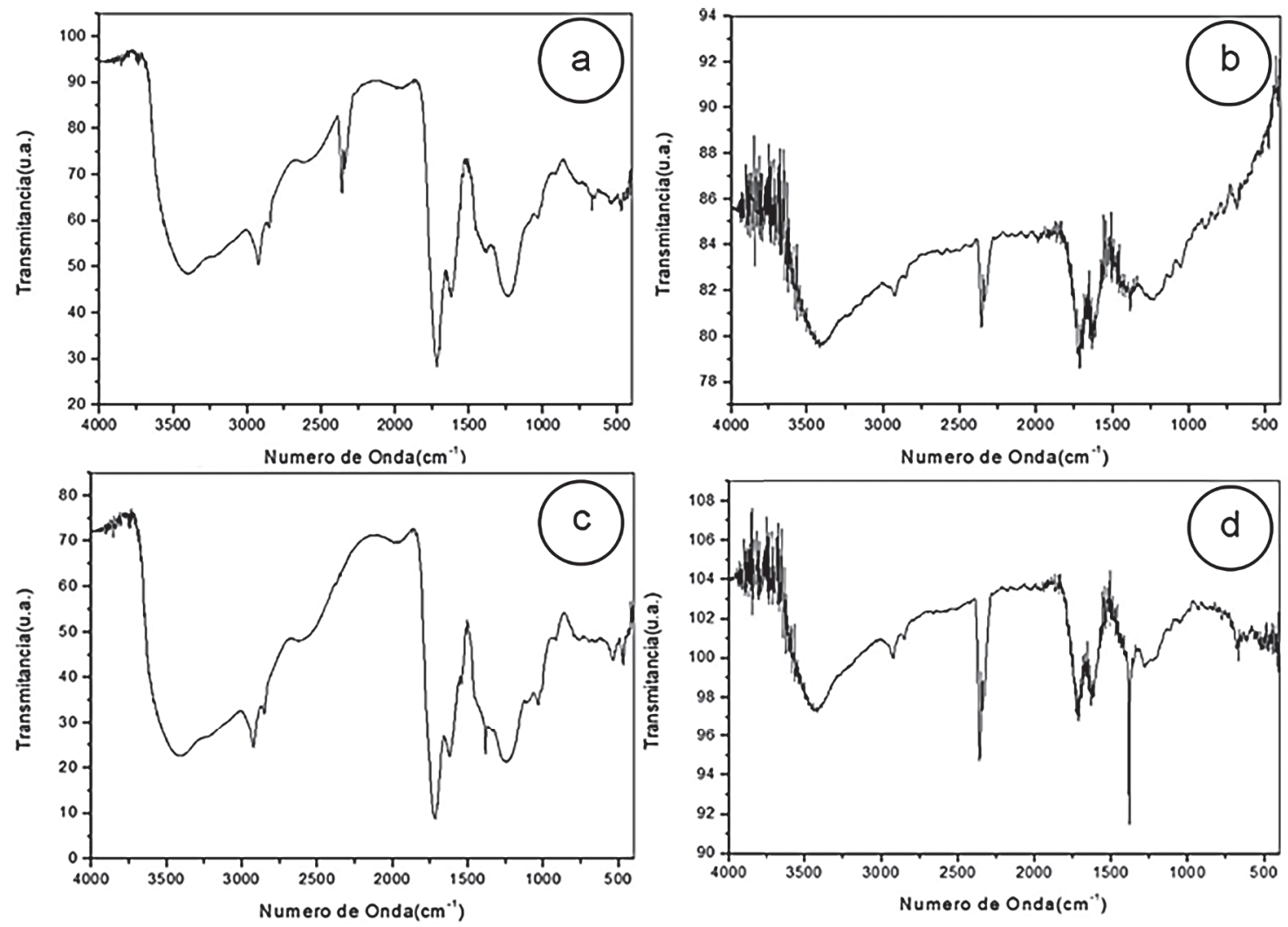

Figura 3. Espectros FTIR de ácidos húmicos extraídos con $\mathrm{H}_{2} \mathrm{O}_{2}$ al 50\%, tiempos de extracción de 3 y 6 horas y tamaños de partículas a malla No. 120 y No. 60. a. Carbón tamizado No.120 oxidado en 3h; b. Carbón tamizado No. 60 oxidado en 3h; c. Carbón tamizado No. 120 oxidado en 6h; d. Carbón tamizado No. 60 oxidado en 6h.
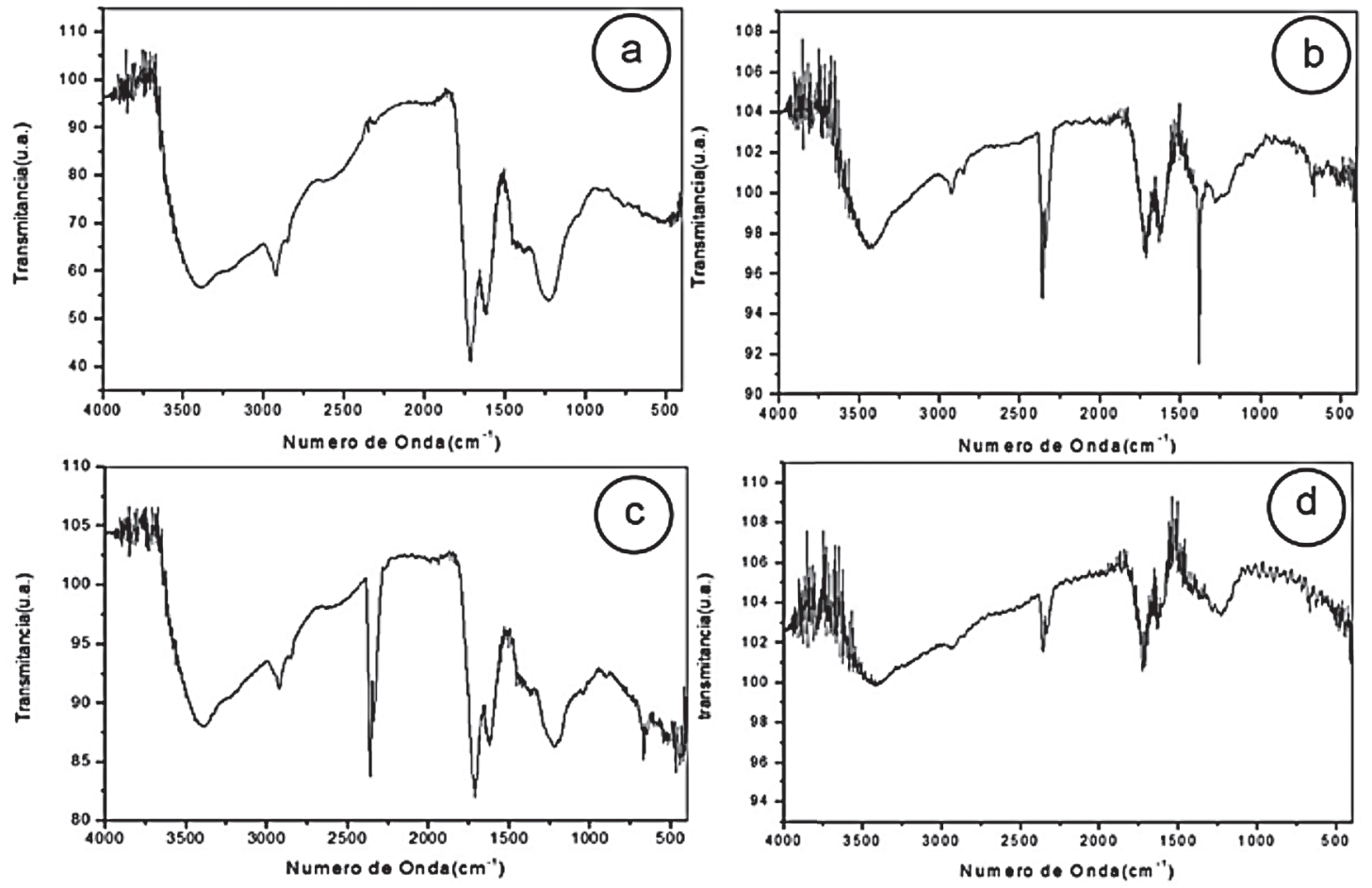

Figura 4. Espectros FTIR de ácidos húmicos extraídos con $\mathrm{H}_{2} \mathrm{O}_{2}$ al $40 \%$, tiempos de extracción de 3 y 6 horas y tamaños de partículas a malla No. 120 y No. 60. a. Carbón tamizado No. 120 oxidado en 3h; b. Carbón tamizado No. 60 oxidado en 3h; c. Carbón tamizado No. 120 oxidado en 6h; d. Carbón tamizado No. 60 oxidado en 6 h. 

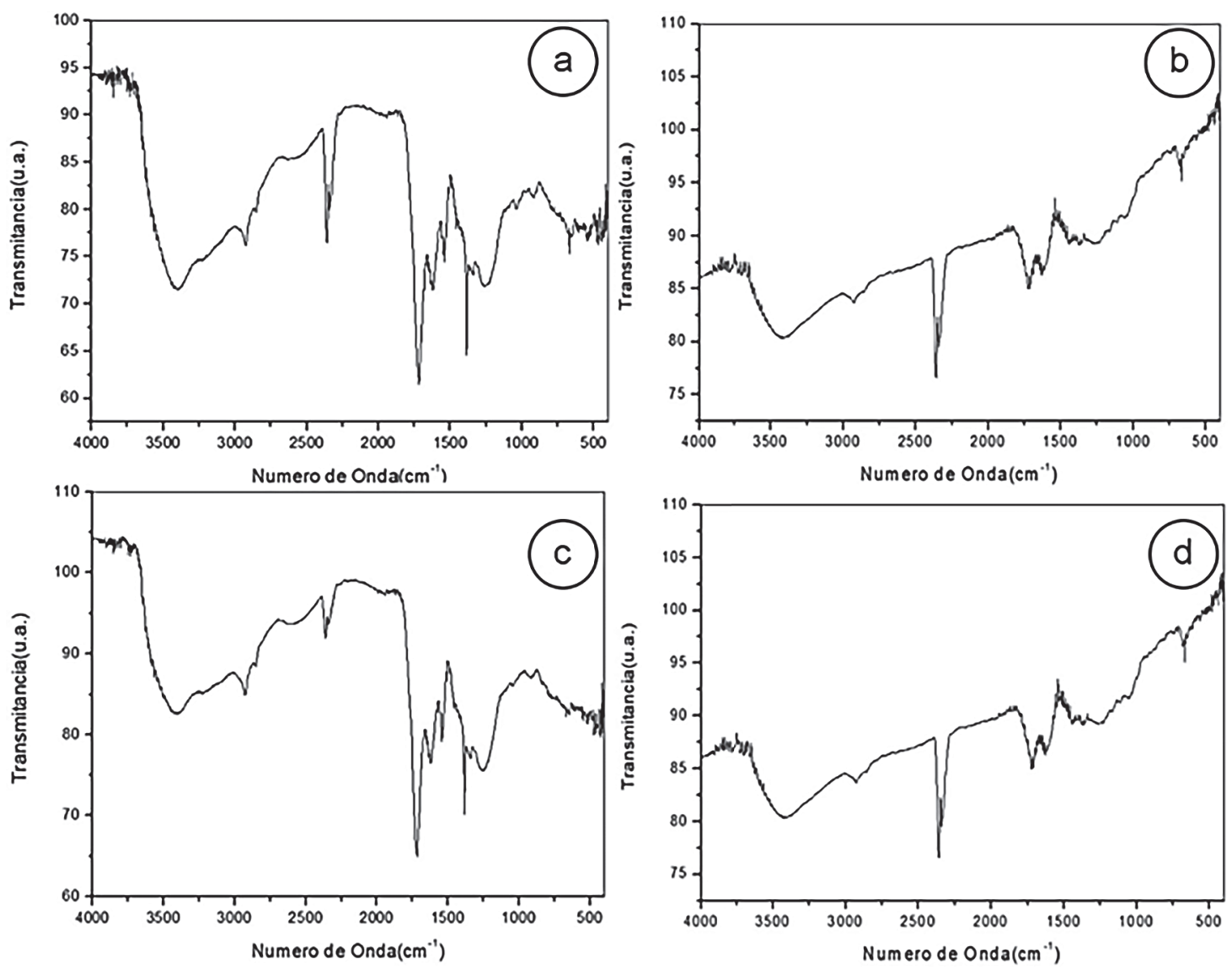

Figura 5. Espectros FTIR de ácidos húmicos extraídos con $\mathrm{H}_{2} \mathrm{O}_{2}$ al 30\%, tiempos de extracción de 3 y 6 horas y tamaños de partículas a malla No. 120 y No. 60. a. Carbón tamizado No. 120 oxidado en 3h; b. Carbón tamizado No. 60 oxidado en 3h; c. Carbón tamizado No. 120 oxidado en 6h; d. Carbón tamizado No. 60 oxidado en 6h.

causarían un impacto ambiental negativo, que se hace necesario resolver, a través de la implementación de un proceso de tratamiento. Una alternativa para mitigar este tipo de daño ambiental sería conducir el efluente, mediante sistema de bombeo a un tanque de almacenamiento, para controlar el $\mathrm{pH}$, posteriormente, someterlo a un proceso de evaporación, por medio de evaporadores de llama directa, hasta llevarlo a concentraciones determinadas, para la recuperación del hidróxido de sodio y ser recirculado nuevamente al proceso.

En el caso del peróxido de hidrógeno, por su gran inestabilidad, se puede descomponer de una manera muy rápida a oxígeno y agua, con posterior liberación de energía.

Los resultados obtenidos, se soportan con los reportados en la literatura científica (De Souza \& Bragança, 2017; Anillo-Correa et al. 2013).

Un gran porcentaje de los ácidos húmicos fueron extraídos del carbón tamizado a malla No. 120, a una concentración de 50\% y durante 6 horas. Los espectros FTIR de los ácidos húmicos que se obtienen, al oxidar con peróxido de hidrógeno, a concentraciones de 50,40 y $30 \%$, confirman que el material obtenido en las oxidaciones son ácidos húmicos, acordes con la estructura macromolecular definida para este tipo de sustancia. El tiempo de extracción y la concentración de agente oxidante utilizado influyen en la cantidad de $\mathrm{AH}$, que puede ser extraído de la muestra carbonácea. El carbón procedente de la mina del cerrejón (Colombia) puede ser un candidato potencial para obtener ácidos húmicos, útiles en la industria agrícola.

Agradecimientos: A la Universidad de Cartagena, por todo el apoyo y la colaboración. Conflictos de intereses: Los autores declaran que no existen conflictos de intereses de ninguna índole.

\section{REFERENCIAS}

1. ANILLO-CORREA, R.; COLPAS-CASTILLO, F.; MEZAFUENTES, E. 2013. Aumento del contenido de ácidos húmicos en un carbón de bajo rango a través de la oxidación con aire y con peróxido de hidrogeno o ácido nítrico. Química Nova. (Brasil). 26(3):387-392. http:// dx.doi.org/10.1590/S0100-40422013000300007

2. AMERICAN SOCIETY FOR TESTING AND MATERIALS ASTM. 2012. Standard Classification of Coals by Rank. (USA). 
3. BARRERA, R.; PÉREZ, J.; SALAZAR, C. 2014. Carbones colombianos: clasificación y caracterización termoquímica para aplicaciones energéticas. Revista Ion. (Colombia). $27(2): 43-54$.

4. CANELLAS, L.P.; OLIVARES, F.L. 2014. Physiological responses to humic substances as plant growth promoter. Chem. Biol. Techno. Agricult. (Italia). 1(1):3. https://doi. org/10.1186/2196-5641-1-3

5. COLPAS CASTILlO, F.; TARÓN DUNOYER, A.; VÁSQUEZ, Y. 2017. Capacidad de adsorción de plomo con intercambiadores catiónicos de carbón xantado. Rev. U.D.C.A Act. \& Div. Cient. (Colombia). 20(2):425-433. https://doi.org/10.31910/rudca.v20.n2.2017.399

6. DE SOUZA, F.; BRAGANÇA, S.R. 2017. Extraction and characterization of humic acid from coal for the application as dispersant of ceramic powders. J. Materials Research and Technology. (Brazil). 6(4):303-310. https://doi. org/10.1016/j.jmrt.2017.08.008

7. FISCHER, T. 2017. Humic supramolecular structures have polar surfaces and unpolar cores in native soil. Chesmosphere. (Reino Unido).183:437-443. https://doi.org/10.1016/j. chemosphere.2017.05.125

8. GOMES DE MELO, B.; LOPES, F.; ANDRADE, M. 2016. Humic acids: Structural properties and multiple functionalities for novel technological developments Materials Science \& Engineering C. (Holanda). 62(1):967974. https://doi.org/10.1016/j.msec.2015.12.001

9. IZQUIERDO, M.; ANTELO, J.; FIO, S.; GONDAR, D.; IGLESIAS, A.; LÓPEZ, R.; ARCE, F. 2004. Caracterización de ácidos fúlvicos y ácidos húmicos extraídos de una turbera minerotrófica. Revista EDAFOLOGIA. (España). 11(3):329-339.

10. KODAMA, R.; SAZAWA, K.; MIYAMOTO, T.; ZHU, Q.; FUKUSHIMA, M. 2018. Potential risk of coupling products between tetrahalobisphenol A and humic acid prepared via oxidation with a biomimetic catalyst. Chemosphere. (Reino Unido) 204:63-70. https://doi. org/10.1016/j.chemosphere.2018.03.141

11. LIPCZYNSKA-KOCHANY, L. 2018. Humic substances, their microbial interactions and effects on biological transformations of organic pollutants in water and soil: A review. Chemosphere. (Reino Unido). 202:420-437. https://doi.org/10.1016/j.chemosphere.2018.03.104

12. LÓPEZ, R.; GONZÁLEZ, G.; VÁZQUEZ, R.; OLIVARES, E.; VIDALE, J.; CARRANZA, R.; ORTEGA, M. 2014.
Metodología para obtener ácidos húmicos y fúlvicos y su caracterización mediante espectrofotometría infrarroja. Rev. Mexicana de Ciencias Agrícolas. 8:1397-1407.

13. MANALS-CUTIÑO, E.; PENEDO, M.; GIRALT-ORTEGA, G. 2011. Thermogravimetric and thermal analysis differential different vegetable biomasses. Tecnología Química. (Cuba). 31(2):180-190.

14. MANZAK, A.; KURŞUN, C.; YILDIZ, Y. 2017. Characterization of humic acid extracted from aqueous solutions with polymer inclusion membranes. J. of the Taiwan Institute of Chemical Engineers. (Taiwan). 81:1420. https://doi.org/10.1016/j.jtice.2017.10.024

15. NARDI, S.; PIZZEGHELLO, D.; SCHIAVON, M.; ERTANI, A. 2016. Plant biostimulants: physiological responses induced by protein hydrolyzed-based products and humic substances in plant metabolism. Scientia Agricola. (Brasil). 73(1):18-23. http://dx.doi.org/10.1590/0103-9016-20150006

16. PANTOJA GUERRA, M.; ALMANZA PÉREZ, Y.; VALERO VALERO, N. 2016. Evaluación del efecto auxin-like de ácidos húmicos en maíz mediante análisis digital de imágenes. Rev. U.D.C.A Act. \& Div. Cient. (Colombia). 19(2):361-369. https://doi.org/10.31910/rudca.v19. n2.2016.90

17. PÉDROT, M.; DIA, A.; DAVRANCHE, M. 2010. Dynamic structure of humic substances: Rare earth elements as a fingerprint. J. Colloid and Interface Science. (USA). 345:206-213. https://doi.org/10.1016/j.jcis.2010.01.069

18. PEÑA, E.M.; HAVEL, J.; PATOČKA, J. 2005. Humic substances-compounds of still unknown structure: applications in agriculture, industry, environment, and biomedicine. J. Appl. Biomed. (República Checa). 3(1):1324.

19. QUINTERO, J.; BONILLA, L. 1991. Obtención de ácidos húmicos por oxidación de carbón con permanganato de potasio y su efecto sobre la disponibilidad de fósforo proveniente de roca fosfórica. Rev. Col. Química. 20(1):914.

20. WANG, S.; LIU, S.; SUN, Y.; JIANG, D.; ZHANG, X. 2017. Investigation of coal components of Late Permian different ranks bark coal using AFM and Micro-FTIR. John and Willie Leone Department of Energy \& Mineral Engineering (EME). (USA). 187(1):51-57. 